Meta

Journal des traducteurs

Translators' Journal

\title{
Stylistique et transformation
}

\section{Jean-Paul Vinay}

Volume 11, numéro 1, mars 1966

URI : https://id.erudit.org/iderudit/004572ar

DOI : https://doi.org/10.7202/004572ar

Aller au sommaire du numéro

Éditeur(s)

Les Presses de l'Université de Montréal

ISSN

0026-0452 (imprimé)

1492-1421 (numérique)

Découvrir la revue

Citer cet article

Vinay, J.-P. (1966). Stylistique et transformation. Meta, 11(1), 3-14.

https://doi.org/10.7202/004572ar

Ce document est protégé par la loi sur le droit d'auteur. L'utilisation des services d'Érudit (y compris la reproduction) est assujettie à sa politique d'utilisation que vous pouvez consulter en ligne.

https://apropos.erudit.org/fr/usagers/politique-dutilisation/
Cet article est diffusé et préservé par Érudit.

Érudit est un consortium interuniversitaire sans but lucratif composé de l’Université de Montréal, l'Université Laval et l'Université du Québec à Montréal. Il a pour mission la promotion et la valorisation de la recherche. https://www.erudit.org/fr/ 


\section{STYLISTIQUE ET TRANSFORMATION}

1. On distingue, depuis Bally, la stylistique interne qui étudie les moyens d'expression d'une langue donnée, et la stylistique externe ou comparée, qui observe les caractères d'une langue tels qu'ils apparaissent par comparaison avec une autre langue (Vinay-Darbelnet, 15, 32). La nature même de la traduction nous pousse tout naturellement vers l'étude de ce deuxième type de stylistique, qui repose d'ailleurs lui-même sur une analyse préalable de la structure grammaticale et stylistique de chacune des langues étudiées ${ }^{1}$.

2. Il n'est peut-être pas inutile de rappeler que la structure d'une langue comprend essentiellement des faits de servitude, alors que la stylistique étudiera les options que cette même structure met à la disposition de l'écrivain. Par exemple, le genre des mots en français est généralement une servitude (la table, le tiroir), mais son emploi peut donner lieu à des options: si la présence de l'article est obligatoire dans Le livre est sur la table, cette même présence devient stylistique devant un nom propre de personne, où il indiquera le dédain ou le mépris: L'empereur aujourd'hui est triste, le Luther lui donne de l'ennui (V. Hugo cité par Grevisse, $\left.\$ 316,1^{\circ}\right)$.

En traduction, la présence de l'article pourra donc être tantôt servitude (La Case de l'Oncle Tom), tantôt option (Guerre et Paix ou La Guerre et la Paix, traduit du russe). Une langue où un tel article serait toujours obligatoire, ou

1. Pour rester sur le plan de la démonstration, point n'est besoin de faire appel à tout l'arsenal des symboles de la logique moderne, dont se servent abondamment les transformationnistes (cf. Bach, 17 sqq.). Qu'il suffise de rappeler que LD signifie « langue de départ» et LA "langue d'arrivée». Ces deux termes traduisent Source language et Target language. Le manque d'imagination lexicologique de la plupart des linguistes européens les pousse à employer " langue source " et " langue cible *.

Les autres symboles utilisés dans le présent article sont: $K$ qui désigne un noyau de transformation $($ Kernel), le signe $=$ qui indique une traduction directe, $\rightarrow$ une transformation ou un passage oblique, $\Rightarrow$ une modulation, $\emptyset$ un signe zéro. Ailleurs, on a utilisé les abréviations ordinaires: $\mathrm{S}$ (sujet), $\mathrm{O}$ (objet), $\mathrm{N}$ (nom), D (déterminant du nom), A (adjectif), V (verbe), C (complément circonstanciel), etc. Les flèches et les lignes indiquent des cheminements et doivent se lire de gauche à droite. 
serait incorporé au substantif qu'il détermine, n'offrirait au traducteur qu'une servitude, par conséquent aucun choix susceptible de déterminer chez le lecteur un effet particulier. J'aime citer à ce propos un livre à «suspense », comme dirait Étiemble, dans lequel le héros, détective privé comme il se doit et grand imbibeur de whisky, rentre chez lui après quelques courses dans le quartier. À la fin du chapitre premier, il ne s'est encore rien passé d'anormal. Nous sommes vaguement inquiets de ce calme insolite dans la vie du héros, quand l'auteur le montre ouvrant la porte de son appartement et allumant l'électricité: Le cadavre était étendu en travers du salon. Ici, nous sommes devant un emploi stylistique de l'article défini. Normalement, le nous renvoie à un fait connu ou à un fait si général que nul n'est censé l'ignorer: Le cheval est la plus noble conquête de l'homme; comme l'auteur ne nous avait pas encore parlé de cadavre, la structure normale (servitude) nous faisait prévoir Un cadavre etc., ce qui est déjà assez saisissant, on l'avouera. Mais l'emploi optionnel de cet article le est infiniment plus saisissant, et devient dès lors pleinement stylistique.

3. Un exercice très utile consiste à rapprocher deux textes ou deux fragments de textes considérés comme rendant compte d'un même message ( $L D=L A$ ), pour étudier leurs ressemblances et leurs divergences. Chaque fois que les unités de traduction de LA seront de même nature que celles de $L D$, on dira que l'on a affaire à une traduction directe ou littérale. Par «même nature », il faut entendre "même espèce » au sens où $\mathrm{M}$. Galichet emploie ce mot. Cette identité peut aller très loin dans l'analyse des morphèmes; par exemple le segment He loved, décomposé en morphèmes successifs $H e-l o v-e d$, peut être rendu en français par Il aimait ou Il aima, c'est-à-dire $I l$ - aim - ait ou $I l$ - aim - $a$, et les deux textes se correspondent de morphème à morphème.

3.1 - Parfois cette correspondance n'est pas linéaire. Que l'on compare $\mathrm{He}$ loved her et Il l'aimait où, par suite d'une servitude syntaxique, les unités her et $l^{\prime}$ ne se situent pas au même endroit de la chaîne parlée ${ }^{2}$. Cependant, la place des unités de traduction n'est pas toujours le résultat d'une servitude.

L'ordre des mots et la démarche d'une langue tendent... à présenter le message selon un certain déroulement, et dans la mesure où ce déroulement est "normal», il ne joue pas de rôle stylistique particulier. Par contre, toute modification du déroulement est susceptible d'attirer l'attention du lecteur, et participe dès lors à la mise en relief. (Vinay-Darbelnet, 211)

C'est ainsi que l'ordre des deux propositions suivantes: (1) On a enrayé le mal et (2) par un traitement énergique, est libre (option) et peut participer à une mise en relief:

A. On a enrayé le mal par un traitement énergique.

B. Par un traitement énergique, on a enrayé le mal.

Même si le choix entre $A$ et $B$ est libre, on remarque que la présence d'une virgule en $\mathrm{B}$ est une servitude qui sert de marque à la distribution particulière de cette phrase (B: 2,1).

2. On aura noté également une perte d'information dans le passage de her à $l$; mais cette perte est le résultat d'une servitude. 
4. Dans la majorité des cas, le rapprochement de LD et de LA révèle d'importantes divergences de structure. Par exemple, la traduction de «He soon realized » par «Il ne tarda pas à se rendre compte » rend le même message avec des moyens différents (Adverbe $\rightarrow$ Locution verbale négativée). On dira que le procédé par lequel un signifié change de catégorie grammaticale est une transposition. Il ne s'agit plus de traduction directe, mais de traduction oblique. (V.-D., 31-39)

5. Si le texte LA révèle au contraire, non plus un changement structural, mais un changement de point de vue, d'éclairage ou de catégorie de pensée, on dira que nous avons affaire à une modulation (V.-D., 75-76 \& 216-229). Par exemple, la traduction de the top floor par le dernier étage comporte une modulation de top (le plus haut) en dernier (d'une série ascendante); le résultat est le même, mais révèle une analyse différente de la réalité. Il en va de même pour praying mantis (mante religieuse), the staggering of holidays (l'étalement des vacances) et, dans un autre ordre d'idées, "He was weighed and found wanting 》 qui se rend normalement par «Il ne fait pas le poids». Il arrive le plus souvent, naturellement, qu'une modulation entraîne aussi une ou plusieurs transpositions (cf. ici weighed/le poids, c'est-à-dire $\mathrm{V} \rightarrow \mathrm{N}$ ).

6. Ces faits ont été rappelés de façon à éclairer la suite de cet article. Je voudrais montrer en effet que la stylistique comparée doit maintenant dépasser le stade du rapprochement et de l'analyse de deux textes, qu'elle doit entamer l'étude des motivations profondes qui poussent les auteurs, écrivant en anglais et en français, à rédiger des textes différents en nature. Il est certes utile de faire remarquer la présence de telle ou telle transposition, de l'enseigner même si ce passage est figé, donc obligatoire (cf. He broke his leg $\rightarrow$ Il $s$ 'est cassé la jambe); mais, particulièrement en matière de segments optionnels et de modulation, il serait bien plus utile de montrer le pourquoi d'une traduction oblique. Jusqu'ici, la stylistique comparée s'est surtout intéressée au comment et particulièrement aux aspects "superficiels » de ce comment: différentes espèces de transpositions et de modulations; déplacement des unités le long de la chaîne parlée; conséquences grammaticales de ces traductions obliques, etc. J'emploie le terme «superficiel » dans le sens où l'entend Chomsky qui oppose surface structure à deep structure (Chomsky, 16 et passim): ce deuxième terme se rendra par «profond» sans difficulté. Un exemple viendra éclairer cette distinction essentielle : considérons le symbole /?/ comme traduisant une motivation profonde de curiosité. Cette pulsion sémantique déterminera en nous un jeu de transformations structurales et aboutira à une question. Celle-ci, en montant à la «surface», c'est-à-dire en se réalisant dans un énoncé écrit ou parlé $E$, prendra différentes formes superficielles $\mathrm{E}_{1}$ et $\mathrm{E}_{2}$ :

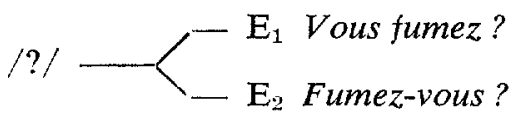

Une chose sera donc de constater qu'en surface, $E_{1}$ et $E_{y}$ se réalisent dans un même contexte ou dans une même situation (ce qui permet d'écrire $E_{1}=E_{2}$ ); 
autre chose, d'évoquer la motivation profonde (A) qui s'est transformée ${ }^{3}$ par des opérations successives (connues hypothétiquement) en énoncés terminaux $\mathrm{E}_{1}$ ou $\mathrm{E}_{2}$. Dans le présent exemple, en simplifiant considérablement la démonstration, on pourra poser des unités profondes appelées noyaux, soit (1) action de fumer, (2) expression d'une curiosité à propos de cette action. Il faudra poser ensuite plusieurs règles pour permettre de transformer ces noyaux profonds en énoncés superficiels; par exemple:

$$
(1) \rightarrow \text { Vous fumez (2) } \rightarrow\left[\begin{array}{l}
\mathrm{E}_{1} \text { Vous fumez? } \\
\mathrm{E}_{2} \text { Fumez-vous? }
\end{array}\right.
$$

Remarquons que la première transformation intéresse surtout le linguiste, de même que la seconde dans la mesure où il ne s'attache pas à la différence entre $E_{1}$ et $E_{2}$. Le traducteur, par contre, voudra savoir pourquoi choisir $E_{2}$ plutôt que $E_{1}$ : le cheminement intérieur ne lui est pas indifférent. On parle bien de «choix», d' « option »: mais y a-t-il vraiment un choix libre? Autrement dit, peut-on envisager une règle de transformation qui s'appliquerait dans certains cas (à définir) et ferait passer automatiquement de Vous fumez à $\mathrm{E}_{1}$ ou à $\mathrm{E}_{2}$ ?

7. Pour bien faire ressortir les deux démarches (superficielle, profonde), je propose d'étudier un exemple pris au hasard dans une circulaire bilingue (anglais/français) adressée aux présidents des différentes sociétés de traducteurs, affiliées à la F.I.T., à l'occasion de la tenue prochaine du $5^{\mathrm{e}}$ congrès de cette fédération qui aura lieu à Lahti (Finlande) du 7 au 13 août 1966.

Les remarques qui vont suivre n'intéressent qu'un court passage de cette circulaire et ne tendent aucunement à critiquer le style des deux textes. On a supposé, pour la commodité de l'exposé, que le texte français était l'aboutissement d'une traduction du texte anglais, mais cette démarche peut être inversée à volonté.

8. Voici les deux textes, reflétant naturellement un même message:

$L D:$ * The brochure of the congress will be distributed to the Translators' Societies in the immediate future, so the participants get more detailed information on the announcement. 》

$L A$ : «Nous vous remettrons ${ }^{4}$ sous peu la brochure du congrès, qui vous fournira les renseignements nécessaires quant à la participation au congrès. »

La première chose à faire est de découper le texte LD en segments appelés UT (unités de traduction, cf. Vinay-Darbelnet; 17-26, et Cahier, exercices 64-66) que nous numéroterons ensuite. Cette numérotation sera alors utilisée

3. Les linguistes américains, après Harris et Chomsky, parlent de «transformational grammar» ou de "generative grammar» (Bach, 176). Les termes "structure superficielle * et " structure profonde " sont employés par Chomsky lui-même dans un article publié dans Diogène 51 (1965): 16. Dans ce même article, cet auteur parle également de grammaire génératrice (p. 20).

4. La variante ferons parvenir, ainsi que d'autres possibilités de traduction par l'actif, seront étudiées plus loin. 
pour étudier les transformations (transpositions, modulations) et les déplacements des UT. L'ordre des UT françaises sera celui où elles se présentent dans le message LA. Ceci permet de figurer les rapprochements par des lignes ou des flèches.

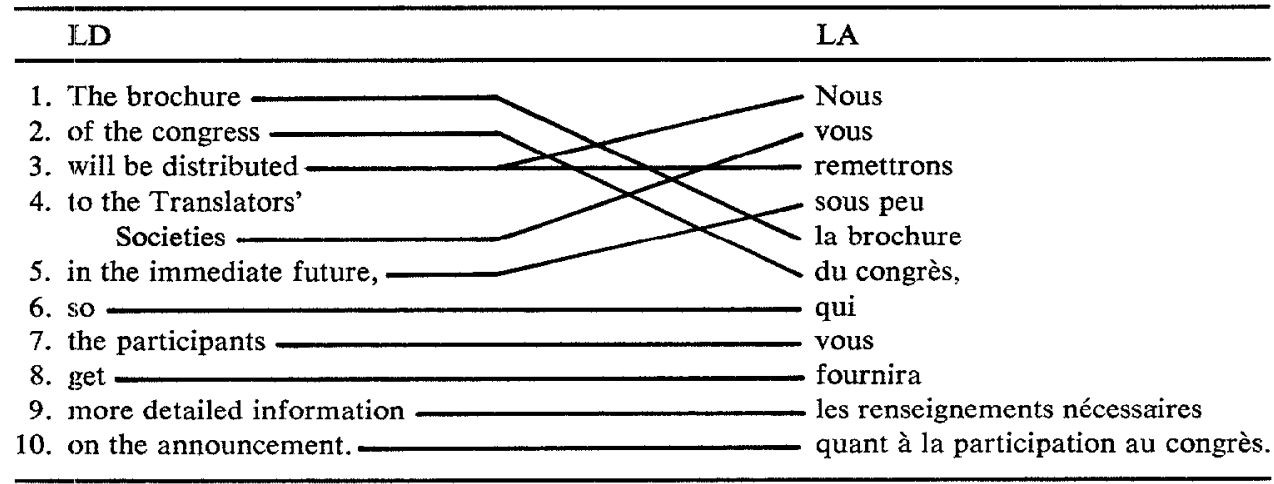

Fig. 1

9. L'analyse des passages schématisés ci-dessus par des traits est généralement conduite, en stylistique comparée, au niveau le plus superficiel, celui des espèces et des catégories grammaticales. C'est ce que fait Barth lorsqu'il présente des statistiques exprimant la fréquence de telle ou telle transposition (Barth, 115-122). C'est également le point de départ des travaux de Bausch et d'Endele, mais il est symptomatique de constater que ces trois auteurs ne se contentent pas d'un classement et d'une description des faits superficiels: ils essaient tous, chacun à sa façon, d'expliquer le pourquoi de ces phénomènes.

Ces passages superficiels peuvent se résumer dans le tableau suivant:

\begin{tabular}{|c|c|c|c|c|c|c|}
\hline 1, 2. DN, PDN & $=$ & DN, PDN & 6. & $\begin{array}{l}\mathrm{C} \\
\mathrm{DN} \text { (nl) }\end{array}$ & $\vec{\rightarrow}$ & Pr, rel. \\
\hline 3. V (f., pass.) & $\Rightarrow$ & Pr., V (f., act.) & 8. & $V(\phi)$ & $\Rightarrow$ & $V$ (sg., f.) \\
\hline 4. PDNN (pl.) & $\rightarrow$ & Pr. (pl.) & 9. & AAN (coll.) & $\rightarrow$ & DNA (pl.) \\
\hline 5. PDAN & $\rightarrow$ & Loc. adv. & 10. & PDN & $\rightarrow$ & Loc. p. DN, PDN \\
\hline
\end{tabular}

Fig. 2

Ce qui se lit « en clair» de la façon suivante:

1,2 -_ (Traduction directe).

3 - Modulation actif/passif; explicitation du sujet.

4 -- Transposition Groupe nominal/Pronom.

5 - Transposition Groupe nominal/Locution adverbiale.

6 - Transposition Conjonction/Pronom relatif.

7 - Transposition Nom/Pronom.

8 - Modulation sens actif/sens passif.

9 - Transposition collectif/pluriel.

10 - Transposition Préposition/Locution prépositive; explicitation du nom.

L'identité symbolique de la transformation 10 (PDN $\rightarrow$ Loc. p., DN, PDN) ne doit pas faire illusion: PDN anglais ne recouvre pas le même segment de message que PDN français. 
On constate donc l'existence de ces passages ou «transformations » d'un genre particulier, mais il manque le fil conducteur. Tout au plus peut-on noter que certains segments sont identiques en nature dans les deux langues et se déroulent de façon parallèle: LD $1,2 \rightarrow$ LA 1,2 ; LD $3,4 \rightarrow$ LA $3,4,3,5$ et surtout LD $6,7,8,9,10 \rightarrow$ LA $6,7,8,9,10$. Des renseignements de ce genre seront indispensables au programmeur qui travaille avec l'ordinateur sur un programme de dépistage de l'information; ils le seront également pour effectuer le programme d'une traduction automatique de l'anglais au français.

10. On doit maintenant se demander par quels cheminements profonds le rédacteur du texte $\mathrm{LD}$ et celui du texte $\mathrm{LA}$ ont passé. La traduction automatique devra absolument se faire une idée sur la nature de ces transformations profondes, et sur leur agencement. Il ne saurait être question d'entrer dans le détail des hypothèses plausibles expliquant l'éclosion des textes de surface: on peut néanmoins en donner les grandes lignes.

L'hypothèse essentielle des tenants de l'école transformationniste réside dans la présence de noyaux de transformation (K) qui se combineront ensuite, s'annuleront ou se remplaceront mutuellement jusqu'à ce que le processus arrive à son terme (segment terminal) qui sera alors, dans le cas d'un ordinateur, imprimé comme texte superficiel ou texte d'aboutissement. Dans le cas présent, l'opération se laisse décomposer comme suit:

$$
\text { Texte LD } \rightarrow \frac{\text { analysé en } \mathrm{K}_{1}, \mathrm{~K}_{2}, \ldots \mathrm{K}_{\mathrm{n}}}{\mathrm{K}_{1}, \mathrm{~K}_{2}, \ldots \mathrm{K}_{\mathrm{n}} \rightarrow \text { transformés en texte LA. }}
$$

Essayons de donner une forme linguistique à ces noyaux $\mathrm{K}$. Ils peuvent être transcrits indifféremment en anglais ou en français, ce qui indique que nous sommes ici sur la plate-forme d'échanges sémiotiques entre les deux langues. Les messages superficiels, par contre, ont figé ces échanges dans des formes incompatibles, et mutuellement inintelligibles.

\begin{tabular}{|l|l|}
\hline \multicolumn{2}{|c|}{ TABLEAU DES NOYAUX DE TRANSFORMATION } \\
\hline $\mathrm{K}_{1}$ Il y a une brochure $\mathrm{B}$ & $\mathrm{K}_{5}$ Le futur est proche \\
$\mathrm{K}_{2}$ Il y a des sociétés STr. & $\mathbf{K}_{6}$ II y a un secrétariat S \\
$\mathrm{K}_{3}$ B passe à STr. (O) & $\mathbf{K}_{7}$ S exécute $\mathrm{O}$ \\
$\mathrm{K}_{4}$ Opération effectuée au futur (f.) & \\
\hline
\end{tabular}

Fig. 3

Nous pouvons donc étudier la distribution des noyaux $\mathrm{K}$ le long de la chaîne parlée dans les deux textes, puis schématiser cette distribution par un tableau: 


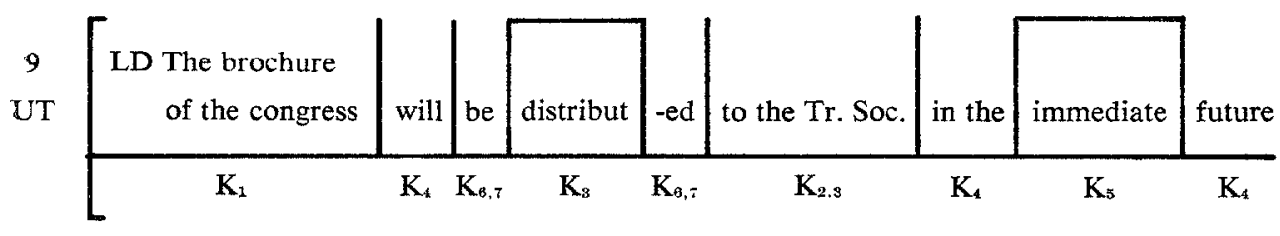

\begin{tabular}{|c|c|c|c|c|c|c|}
\hline \multirow{2}{*}{$\begin{array}{c}6 \\
\text { UT }\end{array}$} & LA Nous & vous & remett- & rons & sous peu & la brochure du congrès \\
\hline & $\mathbf{K}_{8 . \tau}$ & $K_{2,3}$ & $\mathrm{~K}_{3}$ & $\mathbf{K}_{4, \boldsymbol{b}, \boldsymbol{z}}$ & $\mathrm{K}_{5,8}$ & $\mathrm{~K}_{1}$ \\
\hline
\end{tabular}

Fig. 4

À cause de la nature particulière des deux langues rapprochées, l'analyse en UT donne 9 segments en anglais et 6 segments en français. Ces segments rendent compte évidemment de la même réalité $\mathrm{K}$. Voici maintenant un schéma résumant la répartition des éléments $K$ le long de la chaîne parlée de LD et de LA:

L D

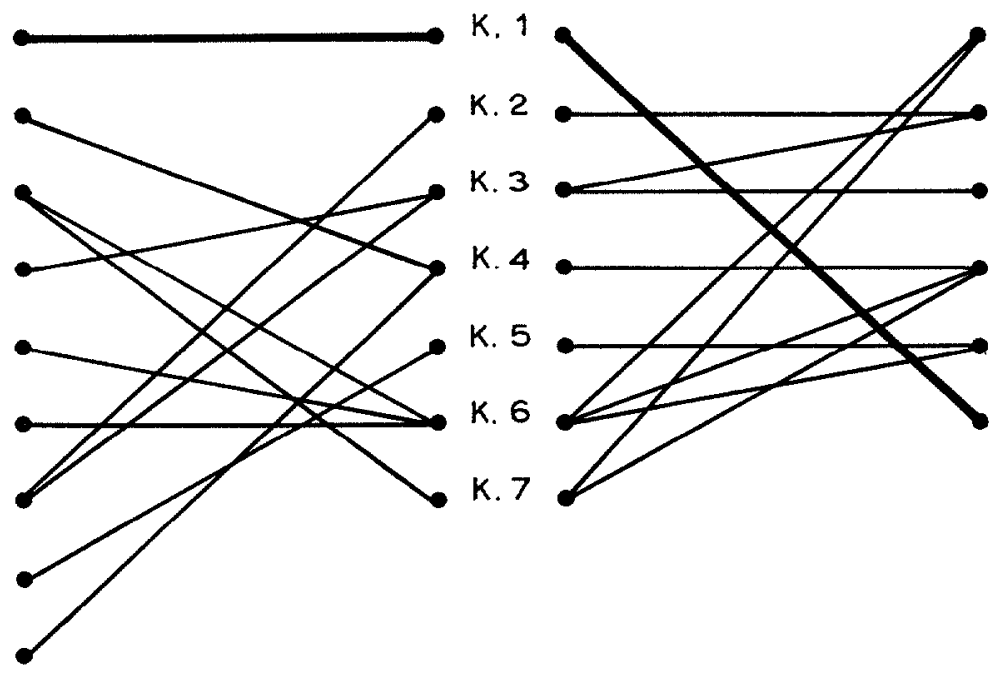

Fig. 5 
Il est remarquable que la pulsion $K_{1}$ (la brochure ủu congrès) se retrouve dans les deux textes en des endroits absolument opposés. La cause est peut-être à chercher dans le système accentuel des deux langues et dans leur déroulement respectif. Ici, le segment mis en relief the brochure of the congress est au début de la phrase (accent tonique principal, note musicale élevée); en français $l a$ brochure du congrès porte un accent fort sur le dernier mot, et un ton descendant qui appelle le point final (ou la virgule, ici).

11. La répartition des noyaux $K$ serait encore plus claire si nous superposions à l'analyse en segments une analyse en fonctions grammaticales. C'est ici qu'apparaît nettement le souci d'aller en profondeur chercher les véritables causes des comportements de surface. L'analyse des UT n'a pas encore été faite en terme de fonctions d'une manière systématique: mais la tendance apparaît chez Bausch (Bausch, 102 par exemple), et surtout chez Endele. C'est là un aboutissement inévitable de l'analyse en profondeur. Donnons-en un rapide exemple:

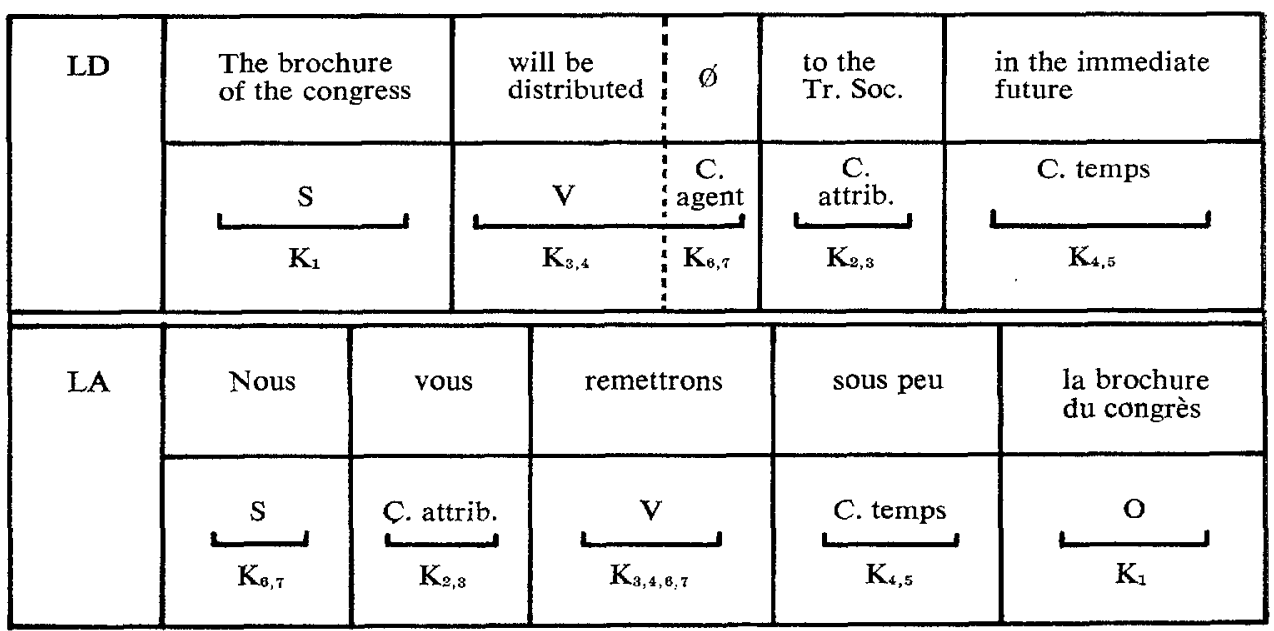

Fig. 6

On remarque immédiatement qu'un segment $\mathbf{K}\left(\mathrm{K}_{6,7}\right)$ se réalise comme $\phi$ en anglais. En effet, dans une construction passive, l'agent peut fort bien être sous-entendu. On ne sait qu'il existe un secrétariat que par le contexte qui précède. Alors qu'en français, l'agent passe au premier plan et se réalise comme un pronom. On peut résumer ce point crucial de la façon suivante:

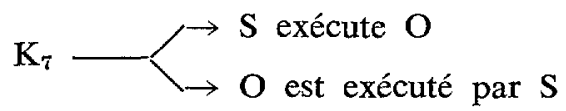

Tout le dilemme est là: si l'on prend la première branche, $\mathrm{K}_{6}$ devient l'exposant de la fonction sujet (modulation), ce qui entraîne l'obligation d'expliciter l'agent (Nous remettrons . . .); $\mathrm{K}_{1}$ prend dès lors la fonction objet. $\mathrm{Si}$, au contraire, 
on opte pour la deuxième branche, $K_{6}$ (l'agent) est inclus dans la formule $K_{7}$ et n'a plus besoin d'être explicité. $K_{1}$ devient le sujet de la phrase passive (The brochure...).

Il est possible de représenter l'ensemble de ces phénomènes par un chassé-croisé (Vinay-Darbelnet, 88) qui partirait d'une explicitation, d'ailleurs possible, de l'agent dans la phrase anglaise. On notera les transformations verticales, qui fonctionnent comme des substituts des segments pleins:

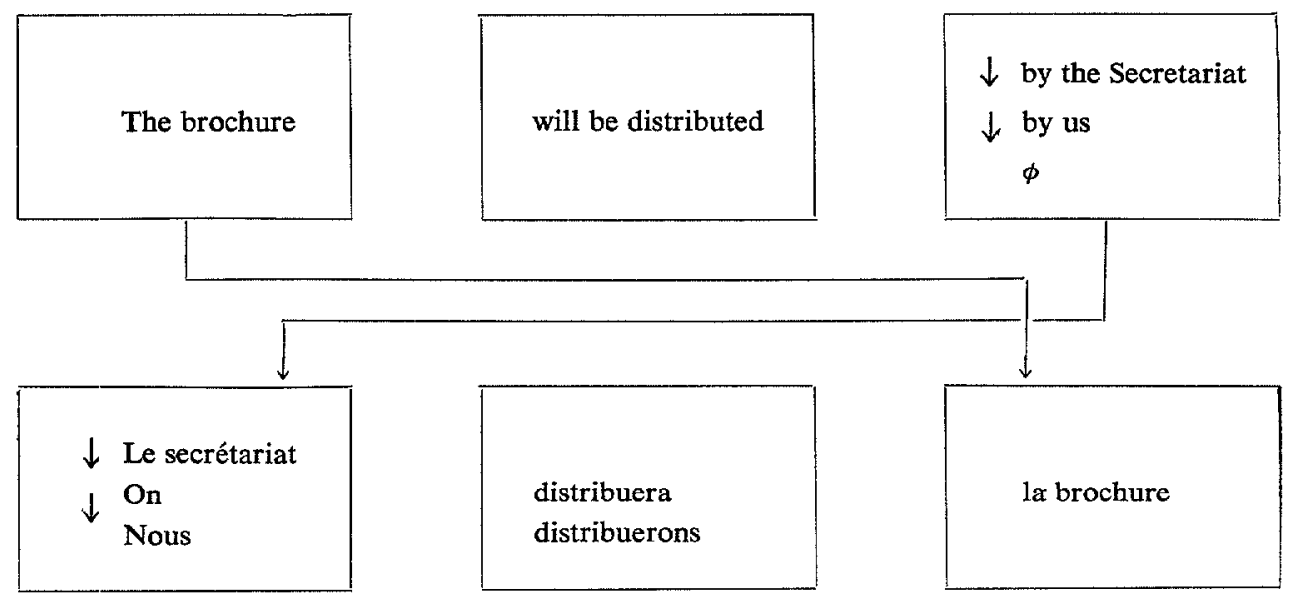

Fig. 7

12. Une étude dans cette direction ferait ressortir les cheminements des transformations effectuées à partir de noyaux profonds $\mathrm{K}$ jusqu'à la surface du texte, tantôt considéré comme une succession de UT, tantôt comme une succession de groupes fonctionnels (les «molécules» de Galichet).

Il resterait cependant une question importante: pourquoi avoir choisi telle branche des dilemmes plutôt que telle autre? Le passif plutôt que l'actif? Les transformationnistes de stricte obédience voudraient bien pouvoir tout mettre en formules, et en vérité, si telle chose était possible, l'avènement de la traduction autornatique ne serait pas très éloigné. Mais, comme le montre bien W. Winter dans un récent article (Language 31.3,1) les choses ne sont pas si simples:

... language is not something developed according to the strict rules of an experiment in a sterile test tube, but the product of many different factors, some in harmony with each other, some independent of each other, some counteracting each other. We should realize that language, a conglomerate of an undetermined number of subsystems, must be very hard, if not impossible, to describe in terms of just one system, however flexible and elaborate... (p. 488)

De sorte que nous nous trouvons devant la possibilité de traduire le texte LD de plusieurs façons; bien plus, la place de certaines UT dans la chaîne parlée pourra varier, et il faudrait étudier de près le résultat de ces variations, qui peuvent apporter de nouvelles valeurs stylistiques ou sémantiques. Je puis proposer notamment la version suivante: 
1. The brochure

La brochure

2. of the congress

du congrès

3. will be distributed

sera distribuée

4. to the Translators' Societies

aux sociétés de traducteurs

5 . in the immediate future, très prochainement,

6. so de sorte que

7. the participants les participants

8. get trouvent

9. more detailed information des renseignements plus complets

10. on the announcement. sur cette manifestation.

Fig. 8

Le lecteur qui voudra bien en faire l'analyse avec les mêmes symboles que ceux utilisés précédemment (fig. 2) se rendra immédiatement compte de la différence entre le nombre et la nature des transpositions des deux versions. Il faudrait mettre au point un barème permettant de juger de la valeur stylistique absolue des UT, ainsi que la valeur du résultat global. Un simple coup d'œil sur le segment 9 (fig. 8) fera ressortir une certaine faiblesse dans l'expression; une série transformationnelle pourrait être prévue, qui effectuerait les changements suivants:

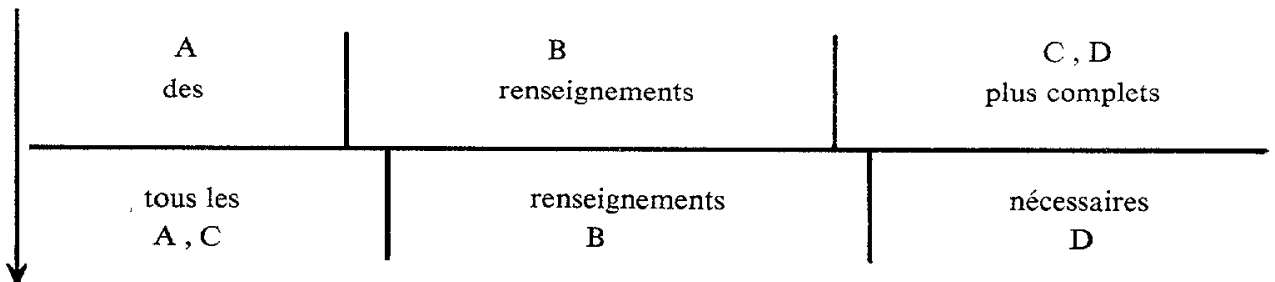

Fig. 9

13. Enfin, il resterait à envisager plusieurs solutions pour chaque version selon la place des segments «sous peu» ou «très prochainement». Il est possible de considérer toutes les réalisations superficielles comme des variantes aléatoires réalisées à partir d'un même schéma de déroulement. Ce serait, très certainement, appauvrir la réalité et nier au traducteur la possibilité de porter des jugements sur la valeur stylistique et sémantique des réalisations de surface. Une étude comparative de toutes ces phrases nous apprendrait peut-être à quelles motivations profondes elles correspondent. Mais nous devons nous rappeler les paroles de W. Winter, citées plus haut: nos préférences iront à telle version parce qu'elle est mieux rythmée, parce qu'elle permet telle mise en relief, ou parce qu'elle correspond à une de nos habitudes d'écriture. Avec le schéma de déroulement donné ci-dessous, le lecteur voudra peut-être s'amuser à construire toutes les versions possibles de Nous vous remettrons sous peu la brochure du congrès. Pour cela, il suffit de remplacer les lettres du diagramme par les segments véritables, selon la clef que voici: 


\begin{tabular}{|c|l|c|l|}
\hline a & nous & $\mathrm{g}$ & la brochure du congrès \\
$\mathrm{b}$ & vous & $\mathrm{h}$ & aux sociétés de traducteurs \\
$\mathrm{c}$ & remettrons & $\mathrm{i}$ & sera \\
$\mathrm{d}$ & ferons parvenir & $\mathrm{j}$ & distribuée \\
$\mathrm{e}$ & sous peu & $\mathrm{k}$ & remise \\
$\mathrm{f}$ & très prochainement & & \\
\hline
\end{tabular}

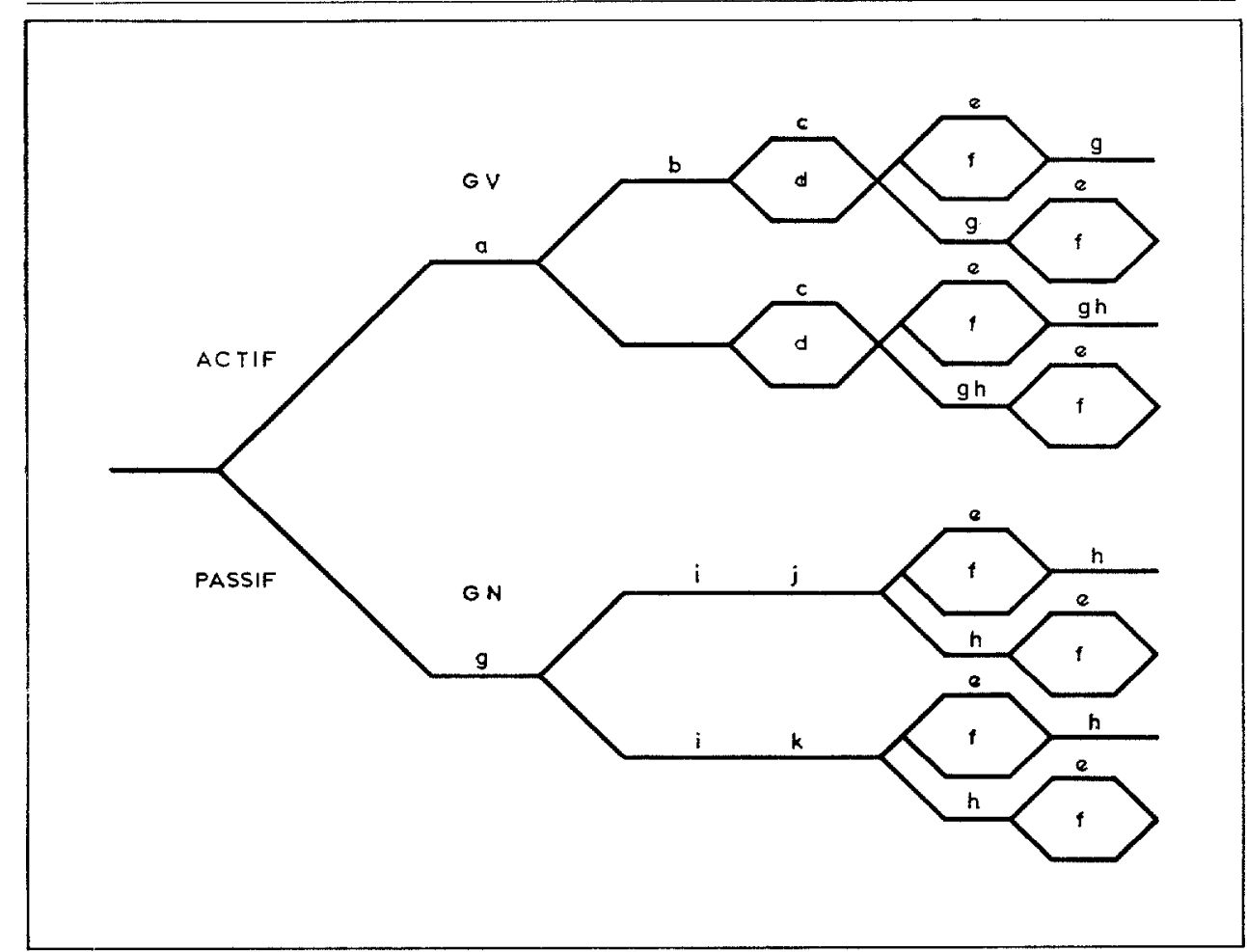

Fig. 10

14. Conclusion: Ce bref exposé a eu pour but de souligner la position dans laquelle se trouve à l'heure actuelle la stylistique comparé ou, si l'on veut, la théorie de la traduction. On peut continuer à travailler sur les réalisations de surface, ce qui présente un intérêt pédagogique certain. On peut, par contre, s'ouvrir un nouveau cheminement vers les motivations profondes de ces réalisations. Tout en croyant que la grammaire transformationnelle n'est pas une panacée pour tous les problèmes de la traduction, j'estime que l'aventure en vaut la peine. Elle devrait nous apporter, chemin faisant, des découvertes inattendues, des vérifications utiles et pourrait bien fournir la réponse à de nombreux problèmes qui assiègent actuellement les spécialistes de la traduction automatique. 


\section{BIBLIOGRAPHIE}

\section{STYLISTIQUE COMPARÉE}

Barth, G., Recherches sur la fréquence et la valeur des parties du discours en français, en anglais et en espagnol. Paris, Didier, 1961.

Bausch, K.-B., Verbum und verbale Periphrase im Französischen und ihre Transposition im Englischen, Deutschen und Spanischen. Tübingen, Eberhard-Karls-Universität, 1963.

Endele, L. A., Wortfolge und Integration. Untersuchungen zum Stil moderner französischer Prosa. Tübingen, Eberhard-Karls-Universität, 1962.

Vinay, J. P. \& Darbelnet, J., Stylistique comparée du français et de l'anglais, nouv. éd., Paris, Didier, 1964: 81-85.

- , Cahier d'exercice $n^{\circ} 1$, nouv. éd., Montréal, Beauchemin, 1965: Exercices 48 et 49 et analyse des textes $\mathrm{B} 1$ à $\mathrm{C} 7$.

\section{LINGUISTIQUE}

Bach, E., * Grammatical Transformations $\gg$, in An Introduction to Transformational Grammars. New York, Holt, 1964: 62-63.

Catford, J. C., "Translation Shifts ", in A Linguistic Theory of Translation. Oxford University Press, 1965: 73-82.

Chomsky, N., "Deep Structures and Grammatical Transformations ", in Aspects of the Theory of Syntax. Cambridge, Mass., M.I.T. Press, 1965: 128-147.

Vinay, J.-P., "La langue neutre et la technique du démontage en traduction », in Stylistique et Linguistique. Montréal, Publications de la Section de Linguistique, II.4 (1956): 46-58.

Winter, W., «Transforms without Kernels ? ", Language 31.3,1 (1965): 484-489.

\section{AUTRES OUVRAGES}

Grevisse, M., Le Bon Usage. Gembloux, Duculot, nouv. éd. 1964.

Shillan, D., Spoken English. London, Longmans Green, 1954, nouv. éd. 1965.

J.-P. V. 